\title{
INVERSE PROBLEM FOR SINGULAR DIFFUSION OPERATOR
}

\author{
ABDULLAH ERGÜN
}

Received 06 June, 2020

\begin{abstract}
In this study, singular diffusion operator with jump conditions is considered. Integral representations have been derived for solutions that satisfy boundary conditions and jump conditions. Some properties of eigenvalues and eigenfunctions are investigated. Asymtotic representation of eigenvalues and eigenfunctions have been obtained. Reconstruction of the singular diffusion operator have been shown by the Weyl function.
\end{abstract}

2010 Mathematics Subject Classification: 34K08; 34L05; 34L10; 34E05

Keywords: inverse problem, Sturm-Liouville, diffusion operator, integral representation

\section{INTRODUCTION}

Let's define the following boundary value problem which will be denoted by $L$ in the sequel all the paper

$$
l(y):=-y^{\prime \prime}+[2 \lambda p(x)+q(x)] y=\lambda^{2} \delta(x) y, x \in[0, \pi] /\left\{p_{1}, p_{2}\right\}
$$

with the boundary conditions

$$
y^{\prime}(0)=0, \quad y(\pi)=0
$$

and the jump conditions

$$
\begin{aligned}
y\left(p_{1}+0\right) & =\alpha_{1} y\left(p_{1}-0\right), \\
y^{\prime}\left(p_{1}+0\right) & =\beta_{1} y^{\prime}\left(p_{1}-0\right)+i \lambda \gamma_{1} y\left(p_{1}-0\right), \\
y\left(p_{2}+0\right) & =\alpha_{2} y\left(p_{2}-0\right), \\
y^{\prime}\left(p_{2}+0\right) & =\beta_{2} y^{\prime}\left(p_{2}-0\right)+i \lambda \gamma_{2} y\left(p_{2}-0\right),
\end{aligned}
$$

where $\lambda$ is a spectral parameter, $q(x) \in L_{2}[0, \pi], p(x) \in W_{2}^{1}[0, \pi], p_{1}, p_{2} \in(0, \pi)$, $p_{1}<p_{2}, \quad\left|\alpha_{1}-1\right|^{2}+\gamma_{1}^{2} \neq 0, \quad\left|\alpha_{2}-1\right|^{2}+\gamma_{2} \neq 0, \quad\left(\beta_{i}=\frac{1}{\alpha_{i}}(i=1,2)\right)$ and $\delta(x)= \begin{cases}1 & x \in\left(0, p_{1}\right) ; \\ \alpha^{2} & x \in\left(p_{1}, p_{2}\right) ; \text { to be } \alpha>0, \alpha \neq 1, \beta>0, \beta \neq 1 \text { real numbers. } \\ \beta^{2} & x \in\left(p_{2}, \pi\right) ;\end{cases}$ 
Direct and inverse problems are important in mathematics, physics and engineering. The inverse problem is called the reconstruction of the operator whose spectral characteristics are given in sequences. For example; to learn the distribution of density in the nonhomogeneous arc according to the wave lengths in mechanics and finding the field potentials according to scattering data in the quantum physics are examples of inverse problems. The first study on inverse problems for differential equations was made by Ambartsumyan in [25]. A significant study in the spectral theory of the singular differential operators was carried out by Levitan in [4]. An important method in the solution of inverse problems is the transformation operators. In [14], Guseinov studied the regular differential equation and the direct spectral problem of the operator under certain initial conditions. In recent years, Weyl function has frequently been used to solve inverse problems. The Weyl function was introduced by $\mathrm{H}$. Weyl in 1910 in the literature. Many studies have been made on direct or inverse problems [1-28]. The solution of discontinuous boundary value problem can be given as an example of concrete problem of mathematical physics. Boundary value problems with discontinuous coefficients are important for applied mathematics and applied sciences.

In [17], Koyunbakan and Panakhov proved that the potential function can be determined on $\left[\frac{\pi}{2}, \pi\right]$ while it is known on $\left[0, \frac{\pi}{2}\right]$ by single spectrum in [12]. In [26], Yang showed that can be determined uniquely diffusion operator from nodal data.

\section{PRELIMINARIES}

Let $\phi(x, \lambda), \psi(x, \lambda)$ be solutions of (1.1) respectively under the boundary conditions

$$
\begin{aligned}
\phi(0, \lambda) & =1, & \phi^{\prime}(0, \lambda) & =0 \\
\psi(\pi, \lambda) & =0, & \psi^{\prime}(\pi, \lambda) & =1
\end{aligned}
$$

and discontinuity conditions $(1.3)-(1.6)$, where $Q(t)=2 \lambda p(t)+q(t)$.

It is obvious that the function $\phi(x, \lambda)$ is similar to [8] satisfies the following integral equations if $0 \leq x<p_{1}$ :

$$
\phi(x, \lambda)=e^{i \lambda x}+\frac{1}{\lambda} \int_{0}^{x} \sin \lambda(x-t) Q(t) y(t, \lambda) d t,
$$

if $p_{1}<x<p_{2}$ :

$$
\begin{aligned}
\phi(x, \lambda)= & \beta_{1}^{+} e^{i \lambda \varsigma^{+}(x)}+\beta_{1}^{-} e^{i \lambda \varsigma^{-}(x)}+\frac{\gamma_{1}}{2 \alpha} e^{i \lambda \varsigma^{+}(x)}-\frac{\gamma_{1}}{2 \alpha} e^{i \lambda \varsigma^{-}(x)} \\
& +\beta_{1}^{+} \int_{0}^{p_{1}} \frac{\sin \lambda\left(\varsigma^{+}(x)-t\right)}{\lambda} J(t) y(t, \lambda) d t \\
& +\beta_{1}^{-} \int_{0}^{p_{1}} \frac{\sin \lambda\left(\varsigma^{-}(x)-t\right)}{\lambda} J(t) y(t, \lambda) d t
\end{aligned}
$$




$$
\begin{aligned}
& -i \frac{\gamma_{1}}{2 \alpha} \int_{0}^{p_{1}} \frac{\cos \lambda\left(\varsigma^{+}(x)-t\right)}{\lambda} J(t) y(t, \lambda) d t \\
& +i \frac{\gamma_{1}}{2 \alpha} \int_{0}^{p_{1}} \frac{\cos \lambda\left(\varsigma^{-}(x)-t\right)}{\lambda} J(t) y(t, \lambda) d t \\
& +\int_{p_{1}}^{x} \frac{\sin \lambda(x-t)}{\lambda} J(t) y(t, \lambda) d t,
\end{aligned}
$$

if $p_{2}<x \leq \pi$ :

$$
\begin{aligned}
\phi(x, \lambda)= & \xi^{+} e^{i \lambda b^{+}(x)}+\xi^{-} e^{i \lambda b^{-}(x)}+\vartheta^{+} e^{i \lambda s^{+}(x)}+\vartheta^{-} e^{i \lambda s^{-}(x)} \\
& +\left(\beta_{1}^{+} \beta_{2}^{+}+\frac{\gamma_{1} \gamma_{2}}{4 \alpha \beta}\right) \int_{0}^{p_{1}} \frac{\sin \lambda\left(b^{+}(x)-t\right)}{\lambda} J(t) y(t, \lambda) d t \\
& +\left(\beta_{1}^{+} \beta_{2}^{-}-\frac{\gamma_{1} \gamma_{2}}{4 \alpha \beta}\right) \int_{0}^{p_{1}} \frac{\sin \lambda\left(s^{+}(x)-t\right)}{\lambda} J(t) y(t, \lambda) d t \\
& +\left(\beta_{1}^{-} \beta_{2}^{-}-\frac{\gamma_{1} \gamma_{2}}{4 \alpha \beta}\right) \int_{0}^{p_{1}} \frac{\sin \lambda\left(b^{-}(x)-t\right)}{\lambda} J(t) y(t, \lambda) d t \\
& +\left(\beta_{1}^{-} \beta_{2}^{+}+\frac{\gamma_{1} \gamma_{2}}{4 \alpha \beta}\right) \int_{p_{1}}^{p_{2}} \frac{\sin \lambda\left(s^{-}(x)-t\right)}{\lambda} J(t) y(t, \lambda) d t \\
& -i\left(\frac{\gamma_{1} \beta_{2}^{+}}{2 \alpha}+\frac{\gamma_{2} \beta_{1}^{+}}{2 \beta}\right) \int_{0}^{p_{1}} \frac{\cos \lambda\left(b^{+}(x)-t\right)}{\lambda} J(t) y(t, \lambda) d t \\
& -i\left(\frac{\gamma_{1} \beta_{2}^{-}}{2 \alpha}-\frac{\gamma_{2} \beta_{1}^{+}}{2 \beta}\right) \int_{0}^{p_{1}} \frac{\cos \lambda\left(s^{+}(x)-t\right)}{\lambda} J(t) y(t, \lambda) d t \\
& +i\left(\frac{\gamma_{1} \beta_{2}^{-}}{2 \alpha}-\frac{\gamma_{2} \beta_{1}^{-}}{2 \beta}\right) \int_{0}^{p_{1}} \frac{\cos \lambda\left(b^{-}(x)-t\right)}{\lambda} J(t) y(t, \lambda) d t \\
& +i\left(\frac{\gamma_{1} \beta_{2}^{+}}{2 \alpha}+\frac{\gamma_{2} \beta_{1}^{-}}{2 \beta}\right) \int_{p_{1}}^{p_{2}} \frac{\cos \lambda\left(s^{-}(x)-t\right)}{\lambda} J(t) y(t, \lambda) d t \\
& +\beta_{2}^{+} \int_{p_{1}}^{p_{2}} \frac{\sin \lambda\left(\beta x-\beta p_{2}+\alpha p_{2}-\alpha t\right)}{\lambda} J(t) y(t, \lambda) d t \\
& -\beta_{2}^{-} \int_{p_{1}}^{p_{2}} \frac{\sin \lambda\left(\beta x-\beta p_{2}-\alpha p_{2}+\alpha t\right)}{\lambda} J(t) y(t, \lambda) d t \\
& -i \frac{\gamma_{2}}{2 \beta} \int_{p_{1}}^{p_{2}} \frac{\cos \lambda\left(\beta x-\beta p_{2}+\alpha p_{2}-\alpha t\right)}{\lambda} J(t) y(t, \lambda) d t \\
& +i \frac{\gamma_{2}}{2 \beta} \int_{p_{1}}^{p_{2}} \frac{\cos \lambda\left(\beta x-\beta p_{2}-\alpha p_{2}+\alpha t\right)}{\lambda} J(t) y(t, \lambda) d t \\
& +\int_{p_{2}}^{x} \frac{\sin \lambda(x-t)}{\lambda} J(t) y(t, \lambda) d t, \\
& \lambda
\end{aligned}
$$




$$
\begin{aligned}
& \phi(x, \lambda)=\xi^{+} e^{i \lambda b^{+}(x)}+\xi^{-} e^{i \lambda b^{-}(x)}+\vartheta^{+} e^{i \lambda s^{+}(x)}+\vartheta^{-} e^{i \lambda s^{-}(x)} \\
& +\left(\beta_{1}^{+} \beta_{2}^{+}+\frac{\gamma_{1} \gamma_{2}}{4 \alpha \beta}\right) \int_{0}^{p_{1}} \frac{\sin \lambda\left(b^{+}(x)-t\right)}{\lambda} J(t) y(t, \lambda) d t \\
& +\left(\beta_{1}^{+} \beta_{2}^{-}-\frac{\gamma_{1} \gamma_{2}}{4 \alpha \beta}\right) \int_{0}^{p_{1}} \frac{\sin \lambda\left(s^{+}(x)-t\right)}{\lambda} J(t) y(t, \lambda) d t \\
& +\left(\beta_{1}^{-} \beta_{2}^{-}-\frac{\gamma_{1} \gamma_{2}}{4 \alpha \beta}\right) \int_{0}^{p_{1}} \frac{\sin \lambda\left(b^{-}(x)-t\right)}{\lambda} J(t) y(t, \lambda) d t \\
& +\left(\beta_{1}^{-} \beta_{2}^{+}+\frac{\gamma_{1} \gamma_{2}}{4 \alpha \beta}\right) \int_{p_{1}}^{p_{2}} \frac{\sin \lambda\left(s^{-}(x)-t\right)}{\lambda} J(t) y(t, \lambda) d t \\
& -i\left(\frac{\gamma_{1} \beta_{2}^{+}}{2 \alpha}+\frac{\gamma_{2} \beta_{1}^{+}}{2 \beta}\right) \int_{0}^{p_{1}} \frac{\cos \lambda\left(b^{+}(x)-t\right)}{\lambda} J(t) y(t, \lambda) d t \\
& -i\left(\frac{\gamma_{1} \beta_{2}^{-}}{2 \alpha}-\frac{\gamma_{2} \beta_{1}^{+}}{2 \beta}\right) \int_{0}^{p_{1}} \frac{\cos \lambda\left(s^{+}(x)-t\right)}{\lambda} J(t) y(t, \lambda) d t \\
& +i\left(\frac{\gamma_{1} \beta_{2}^{-}}{2 \alpha}-\frac{\gamma_{2} \beta_{1}^{-}}{2 \beta}\right) \int_{0}^{p_{1}} \frac{\cos \lambda\left(b^{-}(x)-t\right)}{\lambda} J(t) y(t, \lambda) d t \\
& +i\left(\frac{\gamma_{1} \beta_{2}^{+}}{2 \alpha}+\frac{\gamma_{2} \beta_{1}^{-}}{2 \beta}\right) \int_{p_{1}}^{p_{2}} \frac{\cos \lambda\left(s^{-}(x)-t\right)}{\lambda} J(t) y(t, \lambda) d t \\
& +\beta_{2}^{+} \int_{p_{1}}^{p_{2}} \frac{\sin \lambda\left(\beta x-\beta p_{2}+\alpha p_{2}-\alpha t\right)}{\lambda} J(t) y(t, \lambda) d t \\
& -\beta_{2}^{-} \int_{p_{1}}^{p_{2}} \frac{\sin \lambda\left(\beta x-\beta p_{2}-\alpha p_{2}+\alpha t\right)}{\lambda} J(t) y(t, \lambda) d t \\
& -i \frac{\gamma_{2}}{2 \beta} \int_{p_{1}}^{p_{2}} \frac{\cos \lambda\left(\beta x-\beta p_{2}+\alpha p_{2}-\alpha t\right)}{\lambda} J(t) y(t, \lambda) d t \\
& +i \frac{\gamma_{2}}{2 \beta} \int_{p_{1}}^{p_{2}} \frac{\cos \lambda\left(\beta x-\beta p_{2}-\alpha p_{2}+\alpha t\right)}{\lambda} J(t) y(t, \lambda) d t \\
& +\int_{p_{2}}^{x} \frac{\sin \lambda(x-t)}{\lambda} J(t) y(t, \lambda) d t
\end{aligned}
$$

and it is obvious that the function $\psi(x, \lambda)$ satisfies the following integral equations if $p_{2}<x \leq \pi$ :

$$
\psi(x, \lambda)=\frac{\sin \lambda \beta(x-\pi)}{\lambda \beta}+\int_{x}^{\pi} \frac{\sin \lambda \beta(x-t)}{\lambda \beta} Q(t) y(t, \lambda) d t
$$

if $p_{1}<x<p_{2}$ :

$$
\psi(x, \lambda)=\left(\frac{\alpha \beta_{2}-\gamma_{2}}{2 \alpha \beta_{2} \lambda \alpha_{2} \beta}-\frac{1}{2 \alpha \beta_{2} \lambda}\right) e^{-i \lambda\left(\beta\left(p_{2}-\pi\right)+\alpha\left(p_{2}-x\right)\right)}
$$




$$
\begin{aligned}
& +\left(\frac{\alpha \beta_{2}+\gamma_{2}}{2 \alpha \beta_{2} \lambda \alpha_{2} \beta}+\frac{1}{2 \alpha \beta_{2} \lambda}\right) e^{-i \lambda\left(\beta\left(p_{2}-\pi\right)-\alpha\left(p_{2}-x\right)\right)} \\
& -\left(\frac{\alpha \beta_{2}-\gamma_{2}}{2 \alpha \beta_{2}}-\frac{1}{2}\right) \int_{p_{1}}^{p_{2}} \frac{\sin \lambda\left(x-p_{2}+\alpha t-\alpha p_{2}\right)}{\lambda \alpha} Q(t) y(t, \lambda) d t \\
& +\left(\frac{\alpha \beta_{2}-\gamma_{2}}{2 \alpha \beta_{2}}+\frac{1}{2}\right) \int_{p_{1}}^{p_{2}} \frac{\sin \lambda\left(x-p_{2}-\alpha t+\alpha p_{2}\right)}{\lambda \alpha} Q(t) y(t, \lambda) d t \\
& +\frac{1}{2}\left(\frac{\alpha \beta_{2}-\gamma_{2}}{\alpha \beta_{2} \alpha_{2} \beta}-\frac{1}{\alpha \beta_{2}}\right) \int_{p_{2}}^{\pi} \frac{\sin \lambda\left(x-p_{2}+\beta\left(t-p_{2}\right)\right)}{\lambda \beta} Q(t) y(t, \lambda) d t \\
& -\frac{1}{2}\left(\frac{\alpha \beta_{2}-\gamma_{2}}{\alpha \beta_{2} \alpha_{2} \beta}-\frac{1}{\alpha \beta_{2}}\right) \int_{p_{2}}^{\pi} \frac{\sin \lambda\left(x-p_{2}-\beta\left(t-p_{2}\right)\right)}{\lambda \beta} Q(t) y(t, \lambda) d t \\
& +\frac{\gamma_{2}}{2 \alpha \beta_{2} \lambda} \int_{p_{1}}^{p_{2}} \frac{\cos \lambda\left(x-p_{2}+\alpha t-\alpha p_{2}\right)}{\lambda \alpha} Q(t) y(t, \lambda) d t \\
& -\frac{\gamma_{2}}{2 \alpha \beta_{2} \lambda} \int_{p_{1}}^{p_{2}} \frac{\cos \lambda\left(x-p_{2}-\alpha t+\alpha p_{2}\right)}{\lambda \alpha} Q(t) y(t, \lambda) d t \\
& +\int_{p_{1}}^{x} \frac{\sin \lambda \alpha(x-t)}{\lambda \alpha} Q(t) y(t, \lambda) d t,
\end{aligned}
$$

if $0 \leq x<p_{1}$

$$
\begin{aligned}
\psi(x, \lambda)= & \left(\xi^{+}+\frac{\alpha}{2 \beta_{1}}\right) \eta^{-} e^{-i \lambda\left(b^{-}(\pi)+x\right)}+\left(\xi^{-}-\frac{\alpha}{2 \beta_{1}}\right) \eta^{+} e^{-i \lambda\left(b^{+}(\pi)+x\right)} \\
& +\left(\xi^{-}+\frac{\alpha}{2 \beta_{1}}\right) \eta^{-} e^{-i \lambda\left(s^{+}(\pi)+x\right)}+\left(\xi^{-}-\frac{\alpha}{2 \beta_{1}}\right) \eta^{+} e^{-i \lambda\left(s^{-}(\pi)+x\right)} \\
& +\left(\frac{1}{2 \alpha_{1}}-\frac{\mu^{+}}{4 \beta_{1}}\right) \int_{a_{2}}^{\pi} \frac{\sin \lambda\left(x-p_{2}-\beta t+\beta p_{2}\right)}{\lambda} Q(t) y(t, \lambda) d t \\
& -\left(\frac{1}{2 \alpha_{1}}+\frac{\mu^{+}}{4 \beta_{1}}\right) \int_{p_{2}}^{\pi} \frac{\sin \lambda\left(x-2 p_{1}+p_{2}+\beta t-\beta p_{2}\right)}{\lambda} Q(t) y(t, \lambda) d t \\
& +\left(\frac{1}{2 \alpha_{1}}+\frac{\mu^{-}}{4 \beta_{1}}\right) \int_{p_{2}}^{\pi} \frac{\sin \lambda\left(x-p_{2}-\beta t+\beta p_{2}\right)}{\lambda} Q(t) y(t, \lambda) d t \\
& -\left(\frac{1}{2 \alpha_{1}}-\frac{\mu^{-}}{4 \beta_{1}}\right) \int_{p_{2}}^{\pi} \frac{\sin \lambda\left(x-2 p_{1}+p_{2}-\beta t+\beta p_{2}\right)}{\lambda} Q(t) y(t, \lambda) d t \\
& +\frac{i \gamma_{1}}{2 \alpha_{1} \beta_{1}} \int_{p_{2}}^{\pi} \frac{\cos \lambda\left(x-p_{2}+\beta t-\beta p_{2}\right)}{\lambda} Q(t) y(t, \lambda) d t \\
& -\frac{i \gamma_{1}}{2 \alpha_{1} \beta_{1}} \int_{p_{2}}^{\pi} \frac{\cos \lambda\left(x-2 p_{1}+p_{2}-\beta t+\beta p_{2}\right)}{\lambda} Q(t) y(t, \lambda) d t \\
& -\frac{i \gamma_{1}}{2 \alpha_{1} \beta_{1}} \int_{p_{2}}^{\pi} \frac{\cos \lambda\left(x-p_{2}-\beta t+\beta p_{2}\right)}{\lambda} Q(t) y(t, \lambda) d t
\end{aligned}
$$




$$
\begin{aligned}
& +\frac{i \gamma_{1}}{2 \alpha_{1} \beta_{1}} \int_{p_{2}}^{\pi} \frac{\cos \lambda\left(x-2 p_{1}+p_{2}+\beta t-\beta p_{2}\right)}{\lambda} Q(t) y(t, \lambda) d t \\
& +A \int_{p_{1}}^{p_{2}} \frac{\sin \lambda\left(x-p_{2}+\alpha t-\alpha p_{2}\right)}{\lambda \alpha} Q(t) y(t, \lambda) d t \\
& +A \int_{p_{1}}^{p_{2}} \frac{\sin \lambda\left(x-2 p_{1}+p_{2}-\alpha t+\alpha p_{2}\right)}{\lambda \alpha} Q(t) y(t, \lambda) d t \\
& +B \int_{p_{1}}^{p_{2}} \frac{\cos \lambda\left(x-p_{2}+\alpha t-\alpha p_{2}\right)}{\lambda \alpha} Q(t) y(t, \lambda) d t \\
& +B \int_{p_{1}}^{p_{2}} \frac{\cos \lambda\left(x-2 p_{1}+p_{2}-\alpha t+\alpha p_{2}\right)}{\lambda \alpha} \\
& +C \int_{p_{1}}^{p_{2}} \frac{\sin \lambda\left(x-p_{2}-\alpha t+\alpha p_{2}\right)}{\lambda \alpha} Q(t) y(t, \lambda) d t \\
& +C \int_{p_{1}}^{p_{2}} \frac{\sin \lambda\left(x-2 p_{1}+p_{2}+\alpha t-\alpha p_{2}\right)}{\lambda \alpha} Q(t) y(t, \lambda) d t \\
& +D \int_{p_{1}}^{p_{2}} \frac{\cos \lambda\left(x-p_{2}-\alpha t+\alpha p_{2}\right)}{\lambda \alpha} Q(t) y(t, \lambda) d t \\
& +D \int_{p_{1}}^{p_{2}} \frac{\cos \lambda\left(x-2 p_{1}+p_{2}+\alpha t-\alpha p_{2}\right)}{\lambda \alpha} Q(t) y(t, \lambda) d t \\
& +\int_{0}^{x} \frac{\sin \lambda(x-t)}{\lambda} Q(t) y(t, \lambda) d t,
\end{aligned}
$$

where

$$
\begin{array}{rr}
\varsigma^{ \pm}(x)= \pm \alpha x \mp \alpha p_{1}+p_{1}, & \beta_{1}^{ \pm}=\frac{1}{2}\left(\alpha_{1} \pm \frac{\beta_{1}}{\alpha}\right), \\
b^{ \pm}(x)=\beta x-\beta p_{2}+\mu^{ \pm}\left(p_{2}\right), & s^{ \pm}(x)=-\beta x+\beta p_{2}+\mu^{ \pm}\left(p_{2}\right), \\
\beta_{2}^{\mp}=\frac{1}{2}\left(\alpha_{2} \mp \frac{\alpha \beta_{2}}{\beta}\right), & \xi^{\mp}=\frac{1}{2}\left(\beta_{1}^{\mp} \mp \frac{\gamma_{1}}{2 \alpha}\right)\left(\alpha_{2} \mp \frac{\alpha \beta_{2}}{\beta}+\frac{\gamma_{2}}{\beta}\right), \\
\vartheta^{\mp}=\frac{1}{2}\left(\beta_{1}^{\mp} \frac{\gamma_{1}}{2 \alpha}\right)\left(\alpha_{2} \pm \frac{\alpha \beta_{2}}{\beta}-\frac{\gamma_{2}}{\beta}\right), & \mu^{ \pm}=\left(\frac{\alpha \beta_{2} \pm \gamma_{2}}{2 \alpha \beta_{2} \lambda \alpha_{2} \beta} \pm \frac{1}{2 \alpha \beta_{2} \lambda}\right), \\
A=\left[\left(\frac{i \gamma_{1} \gamma_{2}}{4 \lambda \alpha \alpha_{1} \beta_{1} \beta_{2}}+\left(\frac{-1}{2 \alpha_{1}}-\frac{1}{4 \beta_{1}}\right)\left(\frac{\alpha \beta_{2}-\gamma_{2}}{2 \alpha \beta_{2}}-\frac{1}{2}\right)\right)\right], \\
B=\left[\frac{-i \gamma_{1}}{2 \alpha_{1} \beta_{1}}\left(\frac{\alpha \beta_{2}-\gamma_{2}}{2 \alpha \beta_{2}}-\frac{1}{2}\right)+\frac{1}{2 \alpha_{1}} \frac{\gamma_{2}}{2 \alpha \beta_{2} \lambda}\right], \\
C=\left[\left(\frac{1}{2 \alpha_{1}}+\frac{1}{2 \beta_{1}}\right)\left(\frac{\alpha \beta_{2}-\gamma_{2}}{2 \alpha \beta_{2}}+\frac{1}{2}\right)+\frac{i \gamma_{1} \gamma_{2}}{4 \lambda \alpha \alpha_{1} \beta_{1} \beta_{2}}\right],
\end{array}
$$




$$
D=\left[\frac{i \gamma_{1}}{2 \alpha_{1} \beta_{1}}\left(\frac{\alpha \beta_{2}-\gamma_{2}}{2 \alpha \beta_{2}}+\frac{1}{2}\right)-\frac{\gamma_{2}\left(1-\alpha^{2}\right)}{4 \alpha_{1} \alpha \beta_{2} \lambda}\right] .
$$

Theorem 1. If $p(x) \in W_{2}^{1}(0, \pi)$ and $q(x) \in L_{2}(0, \pi) ; y_{v}(x, \lambda)$ be solutions of $(1.1)$, that satisfies conditions (1.2)-(1.6), has the form

$$
y_{v}(x, \lambda)=y_{0 v}(x, \lambda)+\int_{-x}^{x} K_{v}(x, t) e^{i \lambda t} d t \quad(v=\overline{1,3})
$$

where

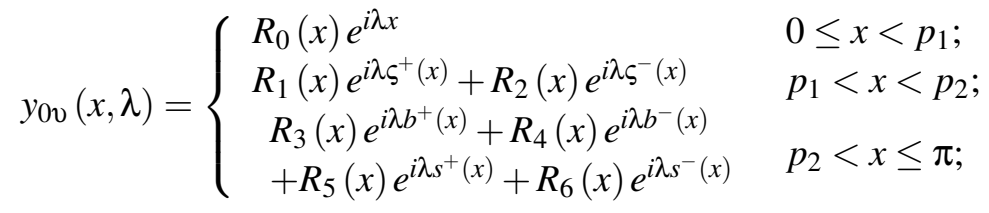

$$
\begin{array}{ll}
R_{0}(x)=e^{-i \int_{0}^{x} p(x) d x}, & R_{1}(x)=\left(\beta_{1}^{+}+\frac{\gamma_{1}}{2 \alpha}\right) R_{0}\left(p_{1}\right) e^{-\frac{i}{\alpha} \int_{p_{1}}^{x} p(t) d t}, \\
R_{2}(x)=\left(\beta_{1}^{-}-\frac{\gamma_{1}}{2 \alpha}\right) R_{0}\left(p_{1}\right) e^{\frac{i}{\alpha} \int_{p_{1}}^{x} p(t) d t}, & R_{3}(x)=\left(\beta_{2}^{+}+\frac{\gamma_{2}}{2 \beta}\right) R_{1}\left(p_{2}\right) e^{-\frac{i}{\beta} \int_{p_{2}}^{x} p(t) d t}, \\
R_{4}(x)=\left(\beta_{2}^{-}+\frac{\gamma_{2}}{2 \beta}\right) R_{2}\left(p_{2}\right) e^{-\frac{i}{\beta} \int_{p_{2}}^{x} p(t) d t}, & R_{5}(x)=\left(\beta_{2}^{-}-\frac{\gamma_{2}}{2 \beta}\right) R_{1}\left(p_{2}\right) e^{\frac{i}{\beta} \int_{p_{2}}^{x} p(t) d t}, \\
R_{6}(x)=\left(\beta_{2}^{+}-\frac{\gamma_{2}}{2 \beta}\right) R_{2}\left(p_{2}\right) e^{\frac{i}{\beta} \int_{p_{2}}^{x} p(t) d t} . &
\end{array}
$$

and $\varpi(x)=\int_{0}^{x}(2|p(t)|+(x-t)|q(t)|) d t$ and the functions $K_{v}(x, t)$ satisfies the inequality

$$
\int_{-x}^{x}\left|K_{v}(x, \lambda)\right| d t \leq e^{c_{v} \varpi(x)}-1
$$

with

$$
\begin{aligned}
c_{1}=1, \quad c_{2} & =\left(\beta_{1}^{+}+\left|\beta_{1}^{-}\right|+\frac{\gamma_{1}}{\alpha}+\frac{2}{\alpha}\right), \\
c_{3} & =\left(\alpha_{2}\left(\beta_{1}^{+}+\left|\beta_{1}^{-}\right|\right)+\frac{1}{\alpha}\left(\beta_{2}^{+}+\left|\beta_{2}^{-}\right|\right)+\frac{\beta^{+}}{\beta}+\frac{\gamma_{2}}{\beta}\right),
\end{aligned}
$$

where

$$
\begin{aligned}
\varsigma^{ \pm}(x) & = \pm \alpha x \mp \alpha p_{1}+p_{1}, & \beta_{1}^{ \pm} & =\frac{1}{2}\left(\alpha_{1} \pm \frac{\beta_{1}}{\alpha}\right), \\
b^{ \pm}(x) & =\beta x-\beta p_{2}+\varsigma^{ \pm}\left(p_{2}\right), & s^{ \pm}(x) & =-\beta x+\beta p_{2}+\varsigma^{ \pm}\left(p_{2}\right), \\
\beta_{2}^{\mp} & =\frac{1}{2}\left(\alpha_{2} \mp \frac{\alpha \beta_{2}}{\beta}\right), & \xi^{\mp} & =\frac{1}{2}\left(\beta_{1}^{\mp} \mp \frac{\gamma_{1}}{2 \alpha}\right)\left(\alpha_{2} \mp \frac{\alpha \beta_{2}}{\beta}+\frac{\gamma_{2}}{\beta}\right), \\
\vartheta^{\mp} & =\frac{1}{2}\left(\beta_{1}^{\mp} \mp \frac{\gamma_{1}}{2 \alpha}\right)\left(\alpha_{2} \pm \frac{\alpha \beta_{2}}{\beta}-\frac{\gamma_{2}}{\beta}\right), & \beta^{ \pm} & =\frac{1}{2}\left(1 \pm \frac{1}{\beta}\right) .
\end{aligned}
$$


The proof is done as in [8].

Theorem 2. Let $p(x) \in W_{2}^{1}(0, \pi)$ and $q(x) \in L_{2}(0, \pi)$. The functions $A(x, t)$, $B(x, t)$, whose first order partial derivatives, are summable on $[0, \pi]$, for each $x \in[0, \pi]$ such that representation

$$
\varphi(x, \lambda)=\varphi_{0}(x, \lambda)+\int_{0}^{x} A(x, t) \cos \lambda t d t+\int_{0}^{x} B(x, t) \sin \lambda t d t
$$

is satisfied.

If $p_{1}<x<p_{2}$ :

$$
\begin{aligned}
\varphi(x, \lambda)= & \left(\beta_{1}^{+}+\frac{\gamma_{1}}{2 \alpha}\right) R_{0}\left(p_{1}\right) \cos \left[\lambda \varsigma^{+}(x)-\frac{1}{\alpha} \int_{p_{1}}^{x} p(t) d t\right] \\
& +\left(\beta_{1}^{-}-\frac{\gamma_{1}}{2 \alpha}\right) R_{0}\left(p_{1}\right) \cos \left[\lambda \varsigma^{-}(x)+\frac{1}{\alpha} \int_{p_{1}}^{x} p(t) d t\right] \\
& +\int_{0}^{\varsigma^{+}(x)} A(x, t) \cos \lambda t d t+\int_{0}^{\varsigma^{+}(x)} B(x, t) \sin \lambda t d t,
\end{aligned}
$$

where $\beta_{1}^{ \pm}=\frac{1}{2}\left(\alpha_{1} \pm \frac{\beta_{1}}{\alpha}\right)$. If $p_{2}<x \leq \pi$ :

$$
\begin{aligned}
\varphi(x, \lambda)= & \left(\beta_{2}^{+}+\frac{\gamma_{2}}{2 \beta}\right) R_{1}\left(p_{2}\right) \cos \left[\lambda b^{+}(x)-\frac{1}{\beta} \int_{p_{2}}^{x} p(t) d t\right] \\
& +\left(\beta_{2}^{-}+\frac{\gamma_{2}}{2 \beta}\right) R_{2}\left(p_{2}\right) \cos \left[\lambda b^{-}(x)-\frac{1}{\beta} \int_{p_{2}}^{x} p(t) d t\right] \\
& +\left(\beta_{2}^{-}-\frac{\gamma_{2}}{2 \beta}\right) R_{1}\left(p_{2}\right) \cos \left[\lambda s^{+}(x)+\frac{1}{\beta} \int_{p_{2}}^{x} p(t) d t\right] \\
& +\left(\beta_{2}^{+}-\frac{\gamma_{2}}{2 \beta}\right) R_{2}\left(p_{2}\right) \cos \left[\lambda s^{-}(x)+\frac{1}{\beta} \int_{p_{2}}^{x} p(t) d t\right] \\
& +\int_{p_{2}}^{x} A(x, t) \cos \lambda t d t+\int_{p_{2}}^{x} B(x, t) \sin \lambda t d t,
\end{aligned}
$$

where $\beta_{2}^{\mp}=\frac{1}{2}\left(\alpha_{2} \mp \frac{\alpha \beta_{2}}{\beta}\right)$. Moreover, the equations

$$
\begin{aligned}
& A\left(x, \varsigma^{+}(x)\right) \cos \frac{\beta(x)}{\alpha}+B\left(x, \varsigma^{+}(x)\right) \sin \frac{\beta(x)}{\alpha} \\
& =\left(\beta_{1}^{+}+\frac{\gamma_{1}}{2 \alpha}\right) \frac{R_{0}\left(p_{1}\right)}{2 \alpha} \int_{0}^{x}\left(q(t)+\frac{p^{2}(t)}{\alpha^{2}}\right) d t \\
& A\left(x, \varsigma^{+}(x)\right) \sin \frac{\beta(x)}{\alpha}-B\left(x, \varsigma^{+}(x)\right) \cos \frac{\beta(x)}{\alpha} \\
& =\left(\beta_{1}^{+}+\frac{\gamma_{1}}{2 \alpha}\right) \frac{R_{0}\left(p_{1}\right)}{2 \alpha^{2}}(p(x)-p(0))
\end{aligned}
$$




$$
\begin{aligned}
& A\left(x, \varsigma^{-}(x)+0\right)-A\left(x, \varsigma^{-}(x)-0\right)=\left(\beta_{1}^{-}-\frac{\gamma_{1}}{2 \alpha}\right) \frac{R_{0}\left(p_{1}\right)}{2 \alpha^{2}} \sin \frac{\beta(x)}{\alpha}(p(x)-p(0)) \\
& +\left(\beta_{1}^{-}-\frac{\gamma_{1}}{2 \alpha}\right) \frac{R_{0}\left(p_{1}\right)}{2 \alpha} \cos \frac{\beta(x)}{\alpha} \int_{0}^{x}\left(q(t)+\frac{p^{2}(t)}{\alpha^{2}}\right) d t \\
& B\left(x, \varsigma^{-}(x)+0\right)-B\left(x, \varsigma^{-}(x)-0\right)=\left(\beta_{1}^{-}-\frac{\gamma_{1}}{2 \alpha}\right) \frac{R_{0}\left(p_{1}\right)}{2 \alpha^{2}} \cos \frac{\beta(x)}{\alpha}(p(x)-p(0)) \\
& -\left(\beta_{1}^{-}-\frac{\gamma_{1}}{2 \alpha}\right) \frac{R_{0}\left(p_{1}\right)}{2 \alpha} \sin \frac{\beta(x)}{\alpha} \int_{0}^{x}\left(q(t)+\frac{p^{2}(t)}{\alpha^{2}}\right) d t \\
& B(x, 0)=\left.\frac{\partial A(x, t)}{\partial t}\right|_{t=0}=0 \\
& A\left(x, s^{-}(x)+0\right)-A\left(x, s^{-}(x)-0\right)=-\left(\beta_{2}^{-}-\frac{\gamma_{2}}{2 \beta}\right) \frac{R_{2}\left(p_{2}\right)}{2 \beta^{2}}(p(x)-p(0)) \sin \frac{\omega(x)}{\beta} \\
& -\left(\beta_{2}^{-}-\frac{\gamma_{2}}{2 \beta}\right) \frac{R_{2}\left(p_{2}\right)}{2 \beta} \int_{0}^{x}\left(q(t)+\frac{p^{2}(t)}{\beta^{2}}\right) d t \cos \frac{\omega(x)}{\beta} \\
& B\left(x, s^{-}(x)+0\right)-B\left(x, s^{-}(x)-0\right)=-\left(\beta_{2}^{-}-\frac{\gamma_{2}}{2 \beta}\right) \frac{R_{2}\left(p_{2}\right)}{2 \beta^{2}}(p(x)-p(0)) \cos \frac{\omega(x)}{\beta} \\
& +\left(\beta_{2}^{-}-\frac{\gamma_{2}}{2 \beta}\right) \frac{R_{2}\left(p_{2}\right)}{2 \beta} \int_{0}^{x}\left(q(t)+\frac{p^{2}(t)}{\beta^{2}}\right) d t \sin \frac{\omega(x)}{\beta} \\
& A\left(x, s^{+}(x)+0\right)-A\left(x, s^{+}(x)-0\right)=-\left(\beta_{2}^{-}-\frac{\gamma_{2}}{2 \beta}\right) \frac{R_{1}\left(p_{2}\right)}{2 \beta^{2}}(p(x)-p(0)) \sin \frac{\omega(x)}{\beta} \\
& -\left(\beta_{2}^{-}-\frac{\gamma_{2}}{2 \beta}\right) \frac{R_{1}\left(p_{2}\right)}{2 \beta} \int_{0}^{x}\left(q(t)+\frac{p^{2}(t)}{\beta^{2}}\right) d t \cos \frac{\omega(x)}{\beta} \\
& B\left(x, s^{+}(x)+0\right)-B\left(x, s^{+}(x)-0\right)=-\left(\beta_{2}^{-}-\frac{\gamma_{2}}{2 \beta}\right) \frac{R_{1}\left(p_{2}\right)}{2 \beta^{2}}(p(x)-p(0)) \cos \frac{\omega(x)}{\beta} \\
& +\left(\beta_{2}^{-}-\frac{\gamma_{2}}{2 \beta}\right) \frac{R_{1}\left(p_{2}\right)}{2 \beta} \int_{0}^{x}\left(q(t)+\frac{p^{2}(t)}{\beta^{2}}\right) d t \sin \frac{\omega(x)}{\beta} \\
& A\left(x, b^{-}(x)+0\right)-A\left(x, b^{-}(x)-0\right)=-\left(\beta_{2}^{-}+\frac{\gamma_{2}}{2 \beta}\right) \frac{R_{2}\left(p_{2}\right)}{2 \beta^{2}}(p(x)-p(0)) \sin \frac{\omega(x)}{\beta} \\
& -\left(\beta_{2}^{-}-\frac{\gamma_{2}}{2 \beta}\right) \frac{R_{2}\left(p_{2}\right)}{2 \beta} \int_{0}^{x}\left(q(t)+\frac{p^{2}(t)}{\beta^{2}}\right) d t \cos \frac{\omega(x)}{\beta}
\end{aligned}
$$




$$
\begin{aligned}
B\left(x, b^{-}(x)+0\right)- & B\left(x, b^{-}(x)-0\right)=\left(\beta_{2}^{-}+\frac{\gamma_{2}}{2 \beta}\right) \frac{R_{2}\left(p_{2}\right)}{2 \beta^{2}}(p(x)-p(0)) \cos \frac{\omega(x)}{\beta} \\
& -\left(\beta_{2}^{-}-\frac{\gamma_{2}}{2 \beta}\right) \frac{R_{2}\left(p_{2}\right)}{2 \beta} \int_{0}^{x}\left(q(t)+\frac{p^{2}(t)}{\beta^{2}}\right) d t \sin \frac{\omega(x)}{\beta}(2.20) \\
A\left(x, b^{+}(x)+0\right)- & A\left(x, b^{+}(x)-0\right)=-\left(\beta_{2}^{+}+\frac{\gamma_{2}}{2 \beta}\right) \frac{R_{1}\left(p_{2}\right)}{2 \beta^{2}}(p(x)-p(0)) \sin \frac{\omega(x)}{\beta} \\
- & \left(\beta_{2}^{+}+\frac{\gamma_{2}}{2 \beta}\right) \frac{R_{1}\left(p_{2}\right)}{2 \beta} \int_{0}^{x}\left(q(t)+\frac{p^{2}(t)}{\beta^{2}}\right) d t \cos \frac{\omega(x)}{\beta} \quad(2.21) \\
B\left(x, b^{+}(x)+0\right)- & B\left(x, b^{+}(x)-0\right)=\left(\beta_{2}^{+}+\frac{\gamma_{2}}{2 \beta}\right) \frac{R_{1}\left(p_{2}\right)}{2 \beta^{2}}(p(x)-p(0)) \cos \frac{\omega(x)}{\beta} \\
- & \left(\beta_{2}^{+}+\frac{\gamma_{2}}{2 \beta}\right) \frac{R_{1}\left(p_{2}\right)}{2 \beta} \int_{0}^{x}\left(q(t)+\frac{p^{2}(t)}{\beta^{2}}\right) d t \sin \frac{\omega(x)}{\beta} \quad(2.22)
\end{aligned}
$$

are held. If in addition we suppose that $p(x) \in W_{2}^{2}(0, \pi), q(x) \in W_{2}^{1}(0, \pi)$, the functions $A(x, t), B(x, t)$ the following system are provided.

$$
\left\{\begin{array}{l}
\frac{\partial^{2} A(x, t)}{\partial x^{2}}-q(x) A(x, t)-2 p(x) \frac{\partial B(x, t)}{\partial t}=\eta \frac{\partial^{2} A(x, t)}{\partial t^{2}} \\
\frac{\partial^{2} B(x, t)}{\partial x^{2}}-q(x) B(x, t)+2 p(x) \frac{\partial A(x, t)}{\partial t}=\eta \frac{\partial^{2} B(x, t)}{\partial t^{2}}
\end{array}\right.
$$

where

$$
\eta= \begin{cases}\alpha^{2} & p_{1}<x<p_{2} \\ \beta^{2} & p_{2}<x<\pi\end{cases}
$$

The proof is done as in [7].

Conversely, if the second order derivatives of functions $A(x, t), B(x, t)$ are summable on $[0, \pi]$ and $A(x, t), B(x, t)$ provides (2.23) system and equations (2.10) (2.22), then the function $\varphi(x, \lambda)$ which is defined by $(1.3)-(1.6)$ is a solution of (1.1) satisfying boundary conditions (1.2).

Definition 1. If there is a nontrivial solution $y_{0}(x)$ that provides the (1.2) conditions for the (1.1) problem, then $\lambda_{0}$ is called eigenvalue. Additionally, $y_{0}(x)$ is called the eigenfunction of the problem corresponding to the eigenvalue $\lambda_{0}$.

Let us assume that $q(x)$ satisfies the following conditions.

$$
\int_{0}^{\pi}\left\{\left|y^{\prime}(x)\right|^{2}+q(x)|y(x)|^{2}\right\} d x>0 .
$$

For all $y(x) \in W_{2}^{2}[0, \pi]$ such that $y(x) \neq 0$ and $y^{\prime}(0) \cdot \overline{y(0)}-y^{\prime}(\pi) \cdot \overline{y(\pi)}=0$.

Lemma 1. The eigenvalues of the boundary value problem $L$ are real. 
Proof. We set $l(y):=\left[-y^{\prime \prime}+q(x) y\right]$. Integration by part yields

$$
(l(y), y)=\int_{0}^{\pi} l(y) \cdot \overline{y(x)} d x=\int_{0}^{\pi}\left\{\left|y^{\prime}(x)\right|^{2}+q(x)|y(x)|^{2}\right\} d x .
$$

Since condition $(2.24)$ holds, it follow that $(l(y), y)>0$.

Lemma 2. Eigenfunction corresponding to different eigenvalues of problem L are orthogonal in the sense of the equality

$$
\left(\lambda_{n}+\lambda_{k}\right) \int_{0}^{\pi} \delta(x) y\left(x, \lambda_{n}\right) y\left(x, \lambda_{k}\right) d x-2 \int_{0}^{\pi} p(x) y\left(x, \lambda_{n}\right) y\left(x, \lambda_{k}\right) d x=0 .
$$

The proof of Lemma 2 carried out as claim [14].

\section{Properties of the SPECTRUM}

Let $\psi(x, \lambda)$ and $\varphi(x, \lambda)$ be any two solutions of equation (1.1),

$$
W[\psi(x, \lambda), \varphi(x, \lambda)]=\psi(x, \lambda) \varphi^{\prime}(x, \lambda)-\psi^{\prime}(x, \lambda) \varphi(x, \lambda),
$$

Wronskian dosen't depend on $x$. In this case, it depends only on the $\lambda$ parameter. Although it is shown as $W[\psi, \varphi]=\Delta(\lambda) . \Delta(\lambda)$ is called the characteristic function of $L$. Clearly, the function $\Delta(\lambda)$ is entire in $\lambda$. It follows that, $\Delta(\lambda)$ has at most a countable set of zeros $\left\{\lambda_{n}\right\}$.

Lemma 3. The zeros $\left\{\lambda_{n}\right\}$ of the characteristic function $\Delta(\lambda)$ coincide with the eigenvalues of the boundary value problem $L$. The functions $\psi\left(x, \lambda_{0}\right)$ and $\varphi\left(x, \lambda_{0}\right)$ are eigenfunctions corresponding to the eigenvalue $\lambda_{n}$, and there exist a sequence $\left(\beta_{n}\right)$ such that

$$
\psi\left(x, \lambda_{n}\right)=\beta_{n} \varphi\left(x, \lambda_{n}\right), \quad \quad \beta_{n} \neq 0 .
$$

The proof of the Lemma 3 is done as in [27].

Let use denote

$$
\alpha_{n}=\int_{0}^{\pi} \delta(x) \varphi^{2}\left(x, \lambda_{n}\right) d x-\frac{1}{\lambda_{n}} \int_{0}^{\pi} p(x) \varphi^{2}\left(x, \lambda_{n}\right) d x, \quad n=1,2,3, \ldots
$$

The numbers $\left\{\alpha_{n}\right\}$ are called normalized numbers of the problem $L$.

Lemma 4. The equality $\dot{\Delta}\left(\lambda_{n}\right)=2 \lambda_{n} \beta_{n} \alpha_{n}$ is obtained. Here $\dot{\Delta}=\frac{d \Delta}{d \lambda}$.

Proof. Since $\varphi(x, \lambda)$ and $\psi(x, \lambda)$ are the solutions of (1.1),

$$
\begin{aligned}
-\varphi^{\prime \prime}(x, \lambda)+[2 \lambda p(x)+q(x)] \varphi(x, \lambda) & =\lambda^{2} \delta(x) \varphi(x, \lambda), \\
-\psi^{\prime \prime}(x, \lambda)+[2 \lambda p(x)+q(x)] \psi(x, \lambda) & =\lambda^{2} \delta(x) \psi(x, \lambda)
\end{aligned}
$$

equations are provided. Hence, we differentiate the equalities with respect to

$$
-\dot{\varphi}^{\prime \prime}(x, \lambda)+[2 \lambda p(x)+q(x)] \dot{\varphi}(x, \lambda)=\lambda^{2} \delta(x) \dot{\varphi}(x, \lambda)+[2 \lambda \delta(x)-2 p(x)] \varphi(x, \lambda),
$$




$$
-\dot{\psi}^{\prime \prime}(x, \lambda)+[2 \lambda p(x)+q(x)] \dot{\psi}(x, \lambda)=\lambda^{2} \delta(x) \dot{\psi}(x, \lambda)+[2 \lambda \delta(x)-2 p(x)] \psi(x, \lambda) .
$$

Thanks to these equations

$$
\begin{aligned}
& \frac{d}{d x}\left\{\varphi(x, \lambda) \cdot \dot{\psi}^{\prime}(x, \lambda)-\varphi^{\prime}(x, \lambda) \cdot \dot{\psi}(x, \lambda)\right\}=-[2 \lambda \delta(x)-2 p(x)] \varphi(x, \lambda) \psi(x, \lambda), \\
& \frac{d}{d x}\left\{\dot{\varphi}(x, \lambda) \cdot \psi^{\prime}(x, \lambda)-\dot{\varphi^{\prime}}(x, \lambda) \cdot \psi(x, \lambda)\right\}=[2 \lambda \delta(x)-2 p(x)] \varphi(x, \lambda) \psi(x, \lambda) .
\end{aligned}
$$

If the last equations are integrated from $x$ to $\pi$ and from 0 to $x$, respectively, by the discontinuity conditions, we obtain

$$
\begin{aligned}
-\left.\left\{\varphi(\xi, \lambda) \cdot \dot{\psi}^{\prime}(\xi, \lambda)-\varphi^{\prime}(\xi, \lambda) \cdot \dot{\psi}(\xi, \lambda)\right\}\right|_{x} ^{\pi} & \\
& =\int_{x}^{\pi}[2 \lambda \delta(\xi)-2 p(\xi)] \varphi(\xi, \lambda) \psi(\xi, \lambda) d \xi
\end{aligned}
$$

and

$\left.\left\{\dot{\varphi}(\xi, \lambda) \cdot \psi^{\prime}(\xi, \lambda)-\dot{\varphi}^{\prime}(\xi, \lambda) \cdot \psi(\xi, \lambda)\right\}\right|_{0} ^{x}=\int_{0}^{x}[2 \lambda \delta(\xi)-2 p(\xi)] \varphi(\xi, \lambda) \psi(\xi, \lambda) d \xi$.

If we add the last equalities side by side, we get

$$
\begin{aligned}
W[\varphi(\xi, \lambda), \dot{\psi}(\xi, \lambda)]+W[\dot{\varphi}(\xi, \lambda), \Psi(\xi, \lambda)] & =-\dot{\Delta}(\lambda) \\
= & \int_{0}^{\pi}[2 \lambda \delta(\xi)-2 p(\xi)] \varphi(\xi, \lambda) \psi(\xi, \lambda) d \xi
\end{aligned}
$$

for $\lambda \rightarrow \lambda_{n}$, this yields

$$
\begin{aligned}
\dot{\Delta}\left(\lambda_{n}\right) & =-\int_{0}^{\pi}\left[2 \lambda_{n} \delta(\xi)-2 p(\xi)\right] \beta_{n} \varphi^{2}\left(\xi, \lambda_{n}\right) d \xi \\
& =2 \lambda_{n} \beta_{n}\left\{\int_{0}^{\pi} \delta(\xi) \varphi^{2}\left(\xi, \lambda_{n}\right) d \xi-\frac{1}{\lambda_{n}} \int_{0}^{\pi} p(\xi) \varphi^{2}\left(\xi, \lambda_{n}\right) d \xi\right\}=2 \lambda_{n} \beta_{n} \alpha_{n} .
\end{aligned}
$$

Denote,

$$
\begin{aligned}
& \Gamma_{n}=\left\{\lambda:|\lambda|=\left|\lambda_{n}^{0}\right|+\delta, \delta>0, n=0,1,2, \ldots\right\}, \\
& G_{n}=\left\{\lambda:\left|\lambda-\lambda_{n}^{0}\right| \geq \delta, \delta>0, n=0,1,2, \ldots\right\},
\end{aligned}
$$

where $\delta$ is sufficiently small positive number. For sufficiently large values of $n$, one has

$$
\left|\Delta(\lambda)-\Delta_{0}(\lambda)\right|<\frac{C_{\delta}}{2} e^{|\tau|\left(\beta \pi-\beta a_{2}+\alpha p_{2}-\alpha p_{1}+p_{1}\right)}, \quad \lambda \in \Gamma_{n} .
$$

As it is shown in [19], $\left|\Delta_{0}(\lambda)\right| \geq C_{\delta} e^{|\operatorname{Im} \lambda| \pi}$ for all $\lambda \in \bar{G}_{\delta}$, where $C_{\delta}>0$

$$
\lim _{|\lambda| \rightarrow \infty} e^{-|\operatorname{Im} \lambda| \pi}\left(\Delta(\lambda)-\Delta_{0}(\lambda)\right)
$$




$$
=\lim _{|\lambda| \rightarrow \infty} e^{-|\operatorname{Im} \lambda| \pi}\left(\int_{0}^{\pi} \tilde{A}(\pi, t) \cos \lambda t d t+\int_{0}^{\pi} \tilde{B}(\pi, t) \sin \lambda t d t\right)=0
$$

is constant. On the other hand, since for sufficiently large values of $n$ (see[23]) we get (3.3). The Lemma 4 is proved.

Lemma 5. The problem $L\left(\alpha, p_{1}, p_{2}\right)$ has countable set of eigenvalues. If one denotes by $\lambda_{1}, \lambda_{2}, \ldots$ the positive eigenvalues arranged in increasing order and by $\lambda_{-1}, \lambda_{-2}, \ldots$ the negative eigenvalues arranged in decreasing order, then eigenvalues of the problem $L\left(\alpha, p_{1}, p_{2}\right)$ have the asymptotic behavior

$$
\lambda_{n}=\lambda_{n}^{0}+\frac{d_{n}}{\lambda_{n}^{0}}+\frac{k_{n}}{\lambda_{n}^{0}} \quad n \rightarrow \infty,
$$

where $k_{n} \in l_{2}, d_{n}$ is a bounded sequence and

$$
\lambda_{n}^{0}=\frac{n \pi}{\beta \pi-\beta p_{2}+\alpha p_{2}-\alpha p_{1}+p_{1}}+\psi_{1}(n) ; \quad \sup _{n}\left|\psi_{1}(n)\right|=c<+\infty .
$$

Proof. According to previous lemma, if $n$ is a sufficiently large natural number and $\lambda \in \Gamma_{n}$, we have $\left|\Delta_{0}(\lambda)\right| \geq C_{\delta} e^{|\operatorname{Im} \lambda| \pi}>\frac{C_{\delta}}{2} e^{|\operatorname{Im} \lambda| \pi}>\left|\Delta(\lambda)-\Delta_{0}(\lambda)\right|$. Applying Rouche's theorem, we conclude that for sufficiently large $n$ inside the contour $\Gamma_{n}$ the functions $\Delta_{0}(\lambda)$ and $\Delta_{0}(\lambda)+\left\{\Delta(\lambda)-\Delta_{0}(\lambda)\right\}=\Delta(\lambda)$ have the same number of zeros. That is, there are exactly $(n+1)$ zeros $\lambda_{1}, \lambda_{2}, \ldots, \lambda_{n}$. Analogously, it is shown by Rouche's theorem that, for sufficiently large values of $n$, the function $\Delta(\lambda)$ has a unique zero inside each circle $\left|\lambda-\lambda_{n}^{0}\right|<\delta$. Since $\delta>0$ is a arbitrary, it follows that $\lambda_{n}=\lambda_{n}^{0}+\varepsilon_{n}$, where $\lim _{n \rightarrow \infty} \varepsilon_{n}=0$. If $\Delta\left(\lambda_{n}\right)=0$, we have

$$
\begin{aligned}
\Delta_{0}\left(\lambda_{n}^{0}+\varepsilon_{n}\right)+ & \int_{0}^{\pi} A(\pi, t) \cos \left(\lambda_{n}^{0}+\varepsilon_{n}\right) t d t+\int_{0}^{\pi} B(\pi, t) \sin \left(\lambda_{n}^{0}+\varepsilon_{n}\right) t d t=0, \\
\Delta_{0}\left(\lambda_{n}^{0}+\varepsilon_{n}\right)= & \left(\beta_{2}^{+}+\frac{\gamma_{2}}{2 \beta}\right) R_{1}\left(p_{2}\right) \cos \left[\left(\lambda_{n}^{0}+\varepsilon_{n}\right) b^{+}(\pi)-\frac{1}{\beta} \int_{p_{2}}^{\pi} p(t) d t\right] \\
& +\left(\beta_{2}^{-}+\frac{\gamma_{2}}{2 \beta}\right) R_{2}\left(p_{2}\right) \cos \left[\left(\lambda_{n}^{0}+\varepsilon_{n}\right) b^{-}(\pi)-\frac{1}{\beta} \int_{p_{2}}^{\pi} p(t) d t\right] \\
& +\left(\beta_{2}^{-}-\frac{\gamma_{2}}{2 \beta}\right) R_{1}\left(p_{2}\right) \cos \left[\left(\lambda_{n}^{0}+\varepsilon_{n}\right) s^{+}(\pi)+\frac{1}{\beta} \int_{p_{2}}^{\pi} p(t) d t\right] \\
& +\left(\beta_{2}^{+}-\frac{\gamma_{2}}{2 \beta}\right) R_{2}\left(p_{2}\right) \cos \left[\left(\lambda_{n}^{0}+\varepsilon_{n}\right) s^{-}(\pi)+\frac{1}{\beta} \int_{p_{2}}^{\pi} p(t) d t\right] .
\end{aligned}
$$

Since $\Delta_{0}(\lambda)$ is an analytical function,

$$
\Delta_{0}\left(\lambda_{n}^{0}+\varepsilon_{n}\right)=\Delta_{0}\left(\lambda_{n}^{0}\right) \varepsilon_{n}+\underset{0}{\dot{\Delta}}\left(\lambda_{n}^{0}\right) \varepsilon_{n}+\frac{\ddot{\Delta}\left(\lambda_{n}^{0}\right)}{2 !} \varepsilon_{n}^{2}+\ldots, \quad \lim _{n \rightarrow \infty} \varepsilon_{n}=0 .
$$


$\lambda_{n}^{0}$ is the roots of the $\Delta_{0}(\lambda)=0$ equation $\Delta_{0}\left(\lambda_{n}^{0}+\varepsilon_{n}\right)=\left[\underset{0}{\dot{\Delta}}\left(\lambda_{n}^{0}\right)+o(1)\right] \varepsilon_{n}, n \rightarrow \infty$ is provided.

$$
\begin{aligned}
{\left[\begin{array}{c}
\dot{\Delta} \\
0
\end{array}\right.} & \left.\left.\lambda_{n}^{0}\right)+o(1)\right] \varepsilon_{n}+\int_{p_{2}}^{s^{-}(x)-0} A(\pi, t) \cos \left(\lambda_{n}^{0}+\varepsilon_{n}\right) t d t \\
+ & \int_{s^{-}(x)+0}^{s^{+}(x)-0} A(\pi, t) \cos \left(\lambda_{n}^{0}+\varepsilon_{n}\right) t d t+\int_{s^{+}(x)+0}^{b^{-}(x)-0} A(\pi, t) \cos \left(\lambda_{n}^{0}+\varepsilon_{n}\right) t d t \\
+ & \int_{b^{-}(x)+0}^{b^{+}(x)-0} A(\pi, t) \cos \left(\lambda_{n}^{0}+\varepsilon_{n}\right) t d t+\int_{b^{+}(x)+0}^{x} A(\pi, t) \cos \left(\lambda_{n}^{0}+\varepsilon_{n}\right) t d t \\
+ & \int_{p_{2}}^{s^{-}(x)-0} B(\pi, t) \sin \left(\lambda_{n}^{0}+\varepsilon_{n}\right) t d t+\int_{s^{-}(x)+0}^{s^{+}(x)-0} B(\pi, t) \sin \left(\lambda_{n}^{0}+\varepsilon_{n}\right) t d t \\
+ & \int_{s^{+}(x)+0}^{b^{-}(x)-0} B(\pi, t) \sin \left(\lambda_{n}^{0}+\varepsilon_{n}\right) t d t+\int_{b^{-}(x)+0}^{b^{+}(x)-0} B(\pi, t) \sin \left(\lambda_{n}^{0}+\varepsilon_{n}\right) t d t \\
& +\int_{b^{+}(x)+0}^{x} B(\pi, t) \sin \left(\lambda_{n}^{0}+\varepsilon_{n}\right) t d t=0
\end{aligned}
$$

It is easy to see that the function $\Delta_{0}(\lambda)=0$ is type of [16], so there is a $\eta_{\delta}>0$ such that $\left|\underset{0}{\dot{\Delta}}\left(\lambda_{n}^{0}\right)\right| \geq \eta_{\delta}>0$ is satisfied for all $n$. We also have

$$
\lambda_{n}^{0}=\frac{n \pi}{\beta \pi-\beta p_{2}+\alpha p_{2}-\alpha p_{1}+p_{1}}+\psi_{1}(n)
$$

where $\sup \left|\psi_{1}(n)\right|<M$ is for some constant $M>0$ [18]. Further, substituting (3.6) into (3.5) after certain transformations, we reach $\varepsilon_{n} \in l_{2}$. have

Since $\left(\int_{0}^{\pi} A_{t}(\pi, t) \sin \left(\lambda_{n}^{0}+\varepsilon_{n}\right) t d t\right) \in l_{2}$ and $\left(\int_{0}^{\pi} B_{t}(\pi, t) \cos \left(\lambda_{n}^{0}+\varepsilon_{n}\right) t d t\right) \in l_{2}$, we

$$
\begin{aligned}
\varepsilon_{n}= & \frac{1}{2 \lambda_{n}^{0} \Delta_{0}\left(\lambda_{n}^{0}\right)}\left\{\left[\left(\beta_{2}^{-}-\frac{\gamma_{2}}{2 \beta}\right) \frac{R_{2}\left(p_{2}\right)}{2 \beta} \sin \left[\lambda_{n}^{0} s^{-}(\pi)+\frac{\omega(x)}{\beta}\right]\right.\right. \\
& +\left(\beta_{2}^{-}-\frac{\gamma_{2}}{2 \beta}\right) \frac{R_{1}\left(p_{2}\right)}{2 \beta} \sin \left[\lambda_{n}^{0} s^{+}(\pi)+\frac{\omega(x)}{\beta}\right] \\
& +\left(\beta_{2}^{-}-\frac{\gamma_{2}}{2 \beta}\right) \frac{R_{2}\left(p_{2}\right)}{2 \beta} \sin \left[\lambda_{n}^{0} b^{-}(\pi)-\frac{\omega(x)}{\beta}\right] \\
& \left.+\left(\beta_{2}^{+}+\frac{\gamma_{2}}{2 \beta}\right) \frac{R_{1}\left(p_{2}\right)}{2 \beta} \sin \left[\lambda_{n}^{0} b^{+}(\pi)-\frac{\omega(x)}{\beta}\right]\right] \int_{0}^{\pi}\left(q(t)+p^{2}(t)\right) d t \\
& +\left[-\left(\beta_{2}^{-}-\frac{\gamma_{2}}{2 \beta}\right) \frac{R_{2}\left(p_{2}\right)}{2 \beta^{2}} \cos \left[\lambda_{n}^{0} s^{-}(\pi)+\frac{\omega(x)}{\beta}\right]\right.
\end{aligned}
$$




$$
\begin{aligned}
& -\left(\beta_{2}^{-}-\frac{\gamma_{2}}{2 \beta}\right) \frac{R_{1}\left(p_{2}\right)}{2 \beta^{2}} \cos \left[\lambda_{n}^{0} s^{+}(\pi)+\frac{\omega(x)}{\beta}\right] \\
& +\left(\beta_{2}^{-}+\frac{\gamma_{2}}{2 \beta}\right) \frac{R_{2}\left(p_{2}\right)}{2 \beta^{2}} \cos \left[\lambda_{n}^{0} b^{-}(\pi)-\frac{\omega(x)}{\beta}\right] \\
& \left.\left.+\left(\beta_{2}^{+}+\frac{\gamma_{2}}{2 \beta}\right) \frac{R_{1}\left(p_{2}\right)}{2 \beta^{2}} \cos \left[\lambda_{n}^{0} b^{+}(\pi)-\frac{\omega(x)}{\beta}\right]\right][p(\pi)-p(0)]\right\}+\frac{k_{n}}{\lambda_{n}^{0}},
\end{aligned}
$$

where

$$
\begin{aligned}
d_{n}= & \frac{1}{2 \Delta_{0}\left(\lambda_{n}^{0}\right)}\left\{\left[\left(\beta_{2}^{-}-\frac{\gamma_{2}}{2 \beta}\right) \frac{R_{2}\left(p_{2}\right)}{2 \beta} \sin \left[\lambda_{n}^{0} s^{-}(\pi)+\frac{\omega(x)}{\beta}\right]\right.\right. \\
& +\left(\beta_{2}^{-}-\frac{\gamma_{2}}{2 \beta}\right) \frac{R_{1}\left(p_{2}\right)}{2 \beta} \sin \left[\lambda_{n}^{0} s^{+}(\pi)+\frac{\omega(x)}{\beta}\right] \\
& +\left(\beta_{2}^{-}-\frac{\gamma_{2}}{2 \beta}\right) \frac{R_{2}\left(p_{2}\right)}{2 \beta} \sin \left[\lambda_{n}^{0} b^{-}(\pi)-\frac{\omega(x)}{\beta}\right] \\
& \left.+\left(\beta_{2}^{+}+\frac{\gamma_{2}}{2 \beta}\right) \frac{R_{1}\left(p_{2}\right)}{2 \beta} \sin \left[\lambda_{n}^{0} b^{+}(\pi)-\frac{\omega(x)}{\beta}\right]\right] \int_{0}^{\pi}\left(q(t)+p^{2}(t)\right) d t \\
& +\left[-\left(\beta_{2}^{-}-\frac{\gamma_{2}}{2 \beta}\right) \frac{R_{2}\left(p_{2}\right)}{2 \beta^{2}} \cos \left[\lambda_{n}^{0} s^{-}(\pi)+\frac{\omega(x)}{\beta}\right]\right. \\
& -\left(\beta_{2}^{-}-\frac{\gamma_{2}}{2 \beta}\right) \frac{R_{1}\left(p_{2}\right)}{2 \beta^{2}} \cos \left[\lambda_{n}^{0} s^{+}(\pi)+\frac{\omega(x)}{\beta}\right] \\
& +\left(\beta_{2}^{-}+\frac{\gamma_{2}}{2 \beta}\right) \frac{R_{2}\left(p_{2}\right)}{2 \beta^{2}} \cos \left[\lambda_{n}^{0} b^{-}(\pi)-\frac{\omega(x)}{\beta}\right] \\
& \left.\left.+\left(\beta_{2}^{+}+\frac{\gamma_{2}}{2 \beta}\right) \frac{R_{1}\left(p_{2}\right)}{2 \beta^{2}} \cos \left[\lambda_{n}^{0} b^{+}(\pi)-\frac{\omega(x)}{\beta}\right]\right][p(\pi)-p(0)]\right\}
\end{aligned}
$$

is bounded sequence. The proof is completed.

The $\varphi(x, \lambda)$ function is $|\lambda| \rightarrow \infty$ in the region $D=\{\lambda: \arg \lambda \in[\varepsilon, \pi-\varepsilon]\}$ for $x>p_{2}$,

$$
\varphi(x, \lambda)=\frac{1}{2}\left(\beta_{2}^{+}+\frac{\gamma_{2}}{2 \beta}\right) \exp \left(-i\left(\lambda b^{+}(x)-w(x)\right)\right)\left(1+O\left(\frac{1}{\lambda}\right)\right) \quad|\lambda| \rightarrow \infty
$$

it has an asymptotic representation where $w(x)=\int_{p_{2}}^{x} p(t) d t$ and $\beta_{2}^{\mp}=\frac{1}{2}\left(\alpha_{2} \mp \frac{\alpha \beta_{2}}{\beta}\right)$. 


\section{INVERSE PROBLEM}

Let us consider the boundary value problem $\tilde{L}$ :

$$
\tilde{L}:=\left\{\begin{array}{l}
l(y):=-y^{\prime \prime}+[2 \lambda \tilde{p}(x)+\tilde{q}(x)] y=\lambda^{2} \tilde{\delta}(x) y, x \in(0, \pi) \\
y^{\prime}(0)=0, y(\pi)=0 \\
y\left(\tilde{p}_{1}+0\right)=\tilde{\alpha}_{1} y\left(\tilde{p}_{1}-0\right) \\
y^{\prime}\left(\tilde{p}_{1}+0\right)=\tilde{\beta}_{1} y^{\prime}\left(\tilde{p}_{1}-0\right)+i \lambda \tilde{\gamma}_{1} y\left(\tilde{p}_{1}-0\right) \\
y\left(\tilde{p}_{2}+0\right)=\tilde{\alpha}_{2} y\left(\tilde{p}_{2}-0\right) \\
y^{\prime}\left(\tilde{p}_{2}+0\right)=\tilde{\beta}_{2} y^{\prime}\left(\tilde{p}_{2}-0\right)+i \lambda \tilde{\gamma}_{2} y\left(\tilde{p}_{2}-0\right)
\end{array}\right.
$$

Let the function $\Phi(x, \lambda)$ denote solution of (1.1) that satisfy the conditions $\Phi^{\prime}(0)=1, \Phi(\pi)=0$ respectively and jump conditions (1.3) - (1.6). Lets define it as $M(\lambda):=\Phi(0, \lambda)$. The $\Phi(x, \lambda)$ and $M(\lambda)$ functions are called the Weyl solution and the Weyl function, respectively.

$$
\Phi(x, \lambda)=M(\lambda) \cdot \varphi(x, \lambda)+S(x, \lambda) \quad \lambda \neq \lambda_{n}, \quad n=1,2,3, \ldots
$$

is true. Because of $\left.W[\varphi, S]\right|_{x=0}=\varphi(0, \lambda) S^{\prime}(0, \lambda)-\varphi^{\prime}(0, \lambda) S(0, \lambda)=1 \neq 0, \varphi(x, \lambda)$ and $S(x, \lambda)$ solutions are linear independent. When $\psi(x, \lambda)$ is solution $(1.1)$,

$$
\begin{aligned}
\psi(x, \lambda) & =A(\lambda) \varphi(x, \lambda)+B(\lambda) S(x, \lambda), \\
\psi^{\prime}(x, \lambda) & =A(\lambda) \varphi^{\prime}(x, \lambda)+B(\lambda) S^{\prime}(x, \lambda) .
\end{aligned}
$$

Due to boundary conditions, $A(\lambda)=\psi(0, \lambda), B(\lambda)=\psi^{\prime}(0, \lambda)=-\Delta(\lambda)$. Then $\psi(x, \lambda)=\psi(0, \lambda) \varphi(x, \lambda)-\Delta(\lambda) S(x, \lambda)$ is obtained. Hence,

$$
\Phi(x, \lambda):=-\frac{\psi(x, \lambda)}{\Delta(\lambda)}=S(x, \lambda)+M(\lambda) \varphi(x, \lambda), \quad M(\lambda)=-\frac{\psi(0, \lambda)}{\Delta(\lambda)} .
$$

The $M(\lambda)$ function is a meromorphic function.

Theorem 3. If $M(\lambda)=\tilde{M}(\lambda)$, then $L=\tilde{L}$.

Proof. Let us define the matrix $P(x, \lambda)=\left[P_{j, k}(x, \lambda)\right],(j, k=1,2)$ by the formula

$$
P(x, \lambda) \cdot\left(\begin{array}{c}
\widetilde{\varphi}(x, \lambda) \widetilde{\Phi}(x, \lambda) \\
\widetilde{\varphi}^{\prime}(x, \lambda) \widetilde{\Phi}^{\prime}(x, \lambda)
\end{array}\right)=\left(\begin{array}{c}
\varphi(x, \lambda) \Phi(x, \lambda) \\
\varphi^{\prime}(x, \lambda) \Phi^{\prime}(x, \lambda)
\end{array}\right) .
$$

In this case

$$
\begin{aligned}
& P_{11}(x, \lambda)=-\varphi(x, \lambda) \frac{\widetilde{\psi}^{\prime}(x, \lambda)}{\widetilde{\Delta}(\lambda)}+\widetilde{\varphi}^{\prime}(x, \lambda) \frac{\psi(x, \lambda)}{\Delta(\lambda)}, \\
& P_{12}(x, \lambda)=-\widetilde{\varphi}(x, \lambda) \frac{\psi(x, \lambda)}{\Delta(\lambda)}+\varphi(x, \lambda) \frac{\widetilde{\psi}(x, \lambda)}{\widetilde{\Delta}(\lambda)}
\end{aligned}
$$




$$
\begin{aligned}
& P_{21}(x, \lambda)=-\varphi^{\prime}(x, \lambda) \frac{\widetilde{\psi}^{\prime}(x, \lambda)}{\widetilde{\Delta}(\lambda)}-\widetilde{\varphi}^{\prime}(x, \lambda) \frac{\psi^{\prime}(x, \lambda)}{\Delta(\lambda)}, \\
& P_{22}(x, \lambda)=-\widetilde{\varphi}(x, \lambda) \frac{\psi^{\prime}(x, \lambda)}{\Delta(\lambda)}+\varphi^{\prime}(x, \lambda) \frac{\widetilde{\psi}(x, \lambda)}{\widetilde{\Delta}(\lambda)} .
\end{aligned}
$$

Hence,

$$
\begin{aligned}
P_{11}(x, \lambda) & =\varphi(x, \lambda)\left[\widetilde{S}(x, \lambda)+\widetilde{M}(\lambda) \cdot \widetilde{\varphi}^{\prime}(x, \lambda)\right]-\widetilde{\varphi}^{\prime}(x, \lambda)[S(x, \lambda)+M(\lambda) \cdot \varphi(x, \lambda)] \\
& =\varphi(x, \lambda) \widetilde{S}(x, \lambda)-\widetilde{\varphi}^{\prime}(x, \lambda) S(x, \lambda)+[\widetilde{M}(\lambda)-M(\lambda)] \varphi(x, \lambda) \widetilde{\varphi}^{\prime}(x, \lambda), \\
P_{12}(x, \lambda) & =\widetilde{\varphi}(x, \lambda)[S(x, \lambda)+M(\lambda) \cdot \varphi(x, \lambda)]-\varphi(x, \lambda)[\widetilde{S}(x, \lambda)+\widetilde{M}(\lambda) \cdot \widetilde{\varphi}(x, \lambda)] \\
& =\widetilde{\varphi}(x, \lambda) S(x, \lambda)-\varphi(x, \lambda) \widetilde{S}(x, \lambda)+[M(\lambda)-\widetilde{M}(\lambda)] \varphi(x, \lambda) \widetilde{\varphi}(x, \lambda), \\
P_{21}(x, \lambda) & =\varphi^{\prime}(x, \lambda)\left[\widetilde{S}(x, \lambda)+\widetilde{M}(\lambda) \cdot \widetilde{\varphi}^{\prime}(x, \lambda)\right]-\widetilde{\varphi}^{\prime}(x, \lambda)\left[S^{\prime}(x, \lambda)+M(\lambda) \cdot \varphi^{\prime}(x, \lambda)\right] \\
& =\varphi^{\prime}(x, \lambda) \widetilde{S}(x, \lambda)-\widetilde{\varphi}^{\prime}(x, \lambda) S^{\prime}(x, \lambda)+[\widetilde{M}(\lambda)-M(\lambda)] \varphi^{\prime}(x, \lambda) \widetilde{\varphi}^{\prime}(x, \lambda), \\
P_{22}(x, \lambda) & =\widetilde{\varphi}(x, \lambda)\left[S^{\prime}(x, \lambda)+M(\lambda) \cdot \varphi^{\prime}(x, \lambda)\right]+\varphi^{\prime}(x, \lambda)[\widetilde{S}(x, \lambda)+\widetilde{M}(\lambda) \cdot \widetilde{\varphi}(x, \lambda)] \\
& =\varphi^{\prime}(x, \lambda) S^{\prime}(x, \lambda)-\varphi^{\prime}(x, \lambda) \widetilde{S}(x, \lambda)+[M(\lambda)-\widetilde{M}(\lambda)] \varphi^{\prime}(x, \lambda) \widetilde{\varphi}(x, \lambda) .
\end{aligned}
$$

from $M(\lambda) \equiv \widetilde{M}(\lambda)$ :

$$
\begin{aligned}
& P_{11}(x, \lambda)=\varphi(x, \lambda) \widetilde{S}(x, \lambda)-\widetilde{\varphi}^{\prime}(x, \lambda) S(x, \lambda), \\
& P_{12}(x, \lambda)=\widetilde{\varphi}(x, \lambda) S(x, \lambda)-\varphi(x, \lambda) \widetilde{S}(x, \lambda), \\
& P_{21}(x, \lambda)=\varphi^{\prime}(x, \lambda) \widetilde{S}(x, \lambda)-\widetilde{\varphi}^{\prime}(x, \lambda) S^{\prime}(x, \lambda), \\
& P_{22}(x, \lambda)=\varphi^{\prime}(x, \lambda) S^{\prime}(x, \lambda)-\varphi^{\prime}(x, \lambda) \widetilde{S}(x, \lambda)
\end{aligned}
$$

are obtained. When $M(\lambda) \equiv \widetilde{M}(\lambda)$, it is clear that the $P_{j, k}(x, \lambda),(j, k=1,2)$ functions are full functions according to $\lambda$. From (3.3); for $\forall x \in[0, \pi], c_{\delta}, C_{\delta}$ constants that provide $\left|P_{11}(x, \lambda)\right| \leq c_{\delta}$ and $\left|P_{12}(x, \lambda)\right| \leq C_{\delta}$ inequalities can be shown. From the Liouville theorem $P_{11}(x, \lambda) \equiv A(x)$ and $P_{12}(x, \lambda) \equiv 0$. From

$$
\begin{aligned}
\varphi(x, \lambda) \cdot \Phi^{\prime}(x, \lambda)-\widetilde{\varphi}^{\prime}(x, \lambda) \cdot \Phi(x, \lambda) & =A(x), \\
\widetilde{\varphi}(x, \lambda) \cdot \Phi(x, \lambda)-\varphi(x, \lambda) \cdot \widetilde{\Phi}(x, \lambda) & =0 \\
\varphi(x, \lambda)=\widetilde{\varphi}(x, \lambda) \cdot A(x), & \Phi(x, \lambda)=\widetilde{\Phi}(x, \lambda) \cdot A(x)
\end{aligned}
$$

are obtained and

$$
W[\varphi, \Phi]=W\left[\varphi(x, \lambda),-\frac{\psi(x, \lambda)}{\Delta(\lambda)}\right]
$$




$$
\begin{aligned}
& =\frac{1}{\Delta(\lambda)} W[\varphi(x, \lambda),-\psi(0, \lambda) \varphi(x, \lambda)+\Delta(\lambda) S(x, \lambda)] \\
& =-\frac{\psi(0, \lambda)}{\Delta(\lambda)} W[\varphi(x, \lambda), \varphi(x, \lambda)]+W[\varphi(x, \lambda), S(x, \lambda)]=1 .
\end{aligned}
$$

And similarly $W[\widetilde{\varphi}, \widetilde{\Phi}]=1$ is obtained. If this equation is written in place of (4.1),

$$
\begin{aligned}
1=W[\varphi(x, \lambda), \Phi(x, \lambda)]=W[A(x) \widetilde{\varphi}(x, \lambda), A(x) \widetilde{\Phi}(x, \lambda)] \\
=A^{2}(x) W[\widetilde{\varphi}(x, \lambda), \widetilde{\Phi}(x, \lambda)]=A^{2}(x)
\end{aligned}
$$

is obtained.

Therefore, $\left(\beta_{2}^{+}+\frac{\gamma_{2}}{2 \beta}\right) \neq 1 ; p_{1}=\tilde{p}_{1}, p_{2}=\tilde{p}_{2}$. We have $A(x)=1$ from (4.1) $\varphi(x, \lambda) \equiv \tilde{\varphi}(x, \lambda)$ and $\Phi(x, \lambda) \equiv \tilde{\Phi}(x, \lambda)$.

When $\varphi(x, \lambda) \equiv \tilde{\varphi}(x, \lambda)$,

$$
\begin{aligned}
& -\varphi^{\prime \prime}+[2 \lambda p(x)+q(x)] \varphi=\lambda^{2} \delta(x) \varphi \\
& -\varphi^{\prime \prime}+[2 \lambda p(x)+q(x)] \varphi=\lambda^{2} \tilde{\delta}(x) \varphi
\end{aligned}
$$

are obtained.

$$
\left\{\lambda^{2}(\delta(x)-\tilde{\delta}(x))+2 \lambda(p(x)-\tilde{p}(x))+(q(x)-\tilde{q}(x))\right\} \varphi \equiv 0 \quad(\text { for } \forall \lambda)
$$

$\delta(x)=\tilde{\delta}(x), p(x)=\tilde{p}(x)$ and $q(x)=\tilde{q}(x)$ a.e. For every $\lambda$ in discontinuity conditions,

$$
\begin{aligned}
\left(\alpha_{1}-\tilde{\alpha}_{1}\right) \varphi\left(p_{1}-0, \lambda\right) & =0 \\
\left(\beta_{1}-\tilde{\beta}_{1}\right) \varphi^{\prime}\left(p_{1}-0, \lambda\right)+\left(\gamma_{1}-\tilde{\gamma}_{1}\right) \varphi\left(p_{1}-0, \lambda\right) & =0 \\
\left(\alpha_{2}-\tilde{\alpha}_{2}\right) \varphi\left(p_{2}-0, \lambda\right) & =0 \\
\left(\beta_{2}-\tilde{\beta}_{2}\right) \varphi^{\prime}\left(p_{2}-0, \lambda\right)+\left(\gamma_{2}-\tilde{\gamma}_{2}\right) \varphi\left(p_{2}-0, \lambda\right) & =0
\end{aligned}
$$

$\alpha_{1}=\tilde{\alpha}_{1}, \beta_{1}=\tilde{\beta}_{1}, \gamma_{1}=\tilde{\gamma}_{1}$ and $\alpha_{2}=\tilde{\alpha}_{2}, \beta_{2}=\tilde{\beta}_{2}, \gamma_{2}=\tilde{\gamma}_{2}$.

Consequently $L=\tilde{L}$. The proof is completed.

\section{REFERENCES}

[1] V. Ambarzumian, "Uber eine frage der eigenwerttheorie," Zeitschrift fur Physik, vol. 53, no. 9-10, pp. 690-695, sep 1929, doi: 10.1007/bf01330827.

[2] R. K. Amirov and A. A. Nabiev, "Inverse problems for the quadratic pencil of the SturmLiouville equations with impulse," Abstract and Applied Analysis, vol. 2013, pp. 1-10, 2013, doi: 10.1155/2013/361989.

[3] L. Anderson, "Inverse eigenvalue problems with discontiuous coefficients," Inverse Problems, vol. 4, pp. 353-397, 1998. 
[4] G. Borg, "Eine umkehrung der sturm-liouvilleschen eigenwertaufgabe," Acta Mathematica, vol. 78, pp. 1-96, 1946.

[5] R. Carlson, "An inverse spectral problem for Sturm-Liouville operators with discontinuous coefficients," Proceedings of the American Mathematical Society, vol. 120, no. 2, pp. 475-475, feb 1994, doi: 10.1090/s0002-9939-1994-1197532-5.

[6] A. Ergün, "A half-inverse problem for the singular diffusion operator with jump conditions," Miskolc Mathematical Notes, to appear.

[7] A. Ergün and R. Amirov, "Direct and inverse problem for diffusion operator with discontinuoity points," TWMS J. App. Eng. Math., vol. 9, pp. 9-21, 2019.

[8] A. Ergün and R. Amirov, "Properties of the spectrum for the singular diffusion operator," Asian Journal of Mathematics and Computer Research, vol. 26, pp. 74-87, 2019.

[9] G. Freiling and V. Yurko, "Inverse spectral problems for singular non-selfadjoint differential operators with discontinuities in an interior point," Inverse Problems, vol. 18, no. 3, pp. 757-773, apr 2002, doi: 10.1088/0266-5611/18/3/316.

[10] S. Gala, Q. Liu, and M. Ragusa, "A new regularity criterion for the nematic liquid crystal flows," Applicable Analysis, vol. 91, no. 9, pp. 1741-1747, 2012.

[11] S. Gala and M. Ragusa, "Logarithmically improved regularity criterion for the boussinesq equations in besov spaces with negative indices," Applicable Analysis, vol. 95, no. 6, pp. 1271-1279, 2016.

[12] M. Gasymov and G. Guseinov, "Determination of a diffusion operator from spectral data," Akad. Nauk Azerbaidzhanskoi SSR, vol. 37, pp. 19-23, 1981.

[13] G. Guseinov, "On the spectral analysis of a quadratic pencil of Sturm-Liouville operators," Dodlady Akad. Nauk SSSR, vol. 285, pp. 1292-1296, 1985.

[14] G. Guseinov, "Inverse spectral problems for a quadratic pencil of Sturm-Liouville operators on a finite interval," Spectral Theory of Operators and Its Applications, pp. 51-101, 1986.

[15] O. Hald, "Discontinuous inverse eigenvalue problems," Comm. on Pure and Appl. Math, vol. 37, pp. 539-577, 1984.

[16] B. Jdanovich, "Formulae for the zeros of drichlet polynomials and quasi-polynomials," Doklady Akad. Nauk SSSR, vol. 135, pp. 1046-1049, 1960.

[17] H. Koyunbakan and E. S. Panakhov, "Half-inverse problem for diffusion operators on the finite interval," Journal of Mathematical Analysis and Applications, vol. 326, no. 2, pp. 1024-1030, feb 2007, doi: 10.1016/j.jmaa.2006.03.068.

[18] M. Krein and B. Y. Levin, "On entire almost periodic functions of exponential type," Doklady Akad. Nauk SSSR., vol. 64, pp. 285-287, 1949.

[19] B. I. Levin, Entire Functions. MGV, 1971.

[20] B. Levitan, Inverse Sturm-Liouville problems. Netherlands: VNU Science Press., 1987.

[21] B. Levitan and M. Gasymov, "Determination of a differantial equation by two spectra," Uspekhi Mathematicheskikh Nauk., vol. 19, pp. 3-63, 1964.

[22] B. Levitan and I. Sargsyan, Introduction to Spectral Theory. Amer. Math. Soc., 1975.

[23] V. Marchenko, Sturm-Liouville Operators and Applications. AMS Chelsea Publishing, 1986.

[24] E. Maris and S. Goktas, "A study of the uniform convergence of spectral expansions for the continuous functions on a sturm-liouville problem," Miskolc Mathematical Notes, vol. 20, no. 2, pp. 1063-1086, 2019.

[25] M. Nabiev, "The inverse spectral problem for the diffusion operator on an interval," Mathematicheskaya Fizika, Analiz, Geometriya, vol. 11, pp. 302-313, 2004.

[26] C.-F. Yang, "Reconstruction of the diffusion operator from nodal data," Zeitschrift für Naturforschung A, vol. 65, no. 1-2, pp. 100-106, jan 2010, doi: 10.1515/zna-2010-1-211. 
[27] V. A. Yurko, Inverse Spectral Problems for Linear Differential Operators and Their Applications. CRC Press, 2000. [Online]. Available: https://www.ebook.de/de/product/4351615/v_a_yurko_ inverse_spectral_problems_for_linear_differential_operators_and_their_applications.html

[28] V. Yurko, Introduction to the Theory of Inverse Spectral Problems. Fizmatlit. Moscow, 2007.

\section{Author's address}

Abdullah Ergün

Cumhuriyet University, Vocational School of Sivas, Sivas., 58140, Turkey

E-mail address: aergun@cumhuriyet.edu.tr 